\title{
THE NONHOMOGENEITY OF THE $E$-TREE-ANSWER TO A PROBLEM RAISED BY D. JENSEN AND A. EHRENFEUCHT
}

\author{
DIDIER MISERCQUE
}

ABSTRACT. We prove that the ordered system of all $C^{\prime} E P$ 's, under the order "admits embedding in" is not homogeneous. This answers a problem raised in [2].

1. Introduction. We assume familiarity with [2]. $L$ denotes the lattice of all $\forall_{1}$-sentences of Peano Arithmetic (PA) modulo PA. $\alpha, \beta, \gamma, \ldots$ denote elements of $L$ (we often identify $\forall_{1}$-sentences with their equivalence classes). 0 and 1 denote respectively the minimum element and the maximum element of $L$.

By the $E$-tree we mean, the class of all prime filters of $L$ under the partial ordering of reverse inclusion $\supset$. By a $C^{1} E P$ is meant the set of all existential sentences (without parameters) satisfied in some model of PA. The following results are well known (see [2] and [4]).

LEMMA 1.1. $F$ is a prime filter of $L$ iff

$$
\neg(L \backslash F) \text { is a } C^{1} E P \text {. }
$$

This gives an isomorphism between the $E$-tree and the ordered system of all $C^{1} E P$ 's.

LEMMA 1.2. (i) The set of the predecessors of an element of the E-tree is totally ordered.

(ii) The E-tree has a minimum element (i.e. $L \backslash\{0\}$ ) and each of its branches has a maximal element.

Jensen and Ehrenfeucht ask [2, p. 243] whether the $E$-tree is homogeneous in the sense that any pair of nonminimal, nonmaximal elements can be exchanged by an automorphism.

\section{Preliminary results.}

LEMMA 2.1. The E-tree has an element $F$ such that

(i) $F$ is not maximal,

(ii) $F$ is not minimal,

(iii) $F$ has no immediate predecessor,

(iv) if $B$ is any branch of the $E$-tree containing $F$, then $F$ has an immediate successor in $B$.

Received by the editors January $21,1981$.

AMS (MOS) subject classifications (1970). Primary 02H20.

${ }^{1}$ Supported in part by an IRSIA grant. I am grateful to the referee for helpful suggestions. 
Proof. Let $\theta$ be a $\forall_{1}$-sentence independent of PA such that PA $+\neg \theta$ and PA have the same $\forall_{1}$-theorems; (such a formula exists by a result of Kreisel, cf. $\$ 1$ of [1]). We denote by $E_{\theta}$ the class of all prime filters of $L$ containing $\theta$ and ordered by $\supset$. It is easily shown that each branch of $E_{\theta}$ has a maximum element. Therefore $E_{\theta}$ has (at least) one maximal element $F_{\theta}$. We will show that $F_{\theta}$ has the required properties.

(i) Let $I=L \backslash F_{\theta}$, we have $\theta \notin I$. Denote by $T$ the theory PA $+\neg I+\neg \theta . T$ is consistent because if PA $+\neg I+\theta$, then

$$
\begin{array}{ll}
\exists \varphi \in I & \mathrm{PA}+\neg \varphi+\theta, \\
& \mathrm{PA}+\neg \theta+\varphi,
\end{array}
$$

and thus

$$
\operatorname{PA} \varphi \varphi \text {. }
$$

This is impossible because PA $+\neg \varphi$ is consistent. Obviously, the prime filter of all $\forall_{1}$-sentences true in any model of $T$ is properly contained in $F_{\theta}$. So $F_{\theta}$ is not maximal.

(ii) If $F_{\theta}=L \backslash\{0\}$, then $E_{\theta}=\{L \backslash\{0\}\}$ and the only prime filter of $L$ containing $\theta$ is $\forall_{1}(\mathbf{N})$. We infer that PA $+\theta \equiv \mathrm{PA}+(L \backslash\{0\})$. This is impossible because PA $+\theta$ is an R.E. theory and PA $+(L \backslash\{0\})$ is a $\pi_{1}^{0}$-non-R.E. theory.

(iii) Suppose that $F_{\theta}$ has an immediate predecessor $F^{\prime}$. Then

$$
\forall \beta \in L \backslash F_{\theta} \quad \mathrm{PA}+F_{\theta}+\beta \vdash F^{\prime}
$$

(because, if this theory is consistent, the class of all $\forall_{1}$-sentences true in any model of $T$ is a prime filter of $L$ containing properly $F_{\theta}$ and therefore $F^{\prime}$; if $\mathrm{PA}+F_{\theta}+\beta$ is not consistent, the result is obvious). Therefore

$$
\forall \alpha \in F^{\prime} \backslash F_{\theta} \quad \forall \beta \in L \backslash F_{\theta} \quad \exists \gamma \in F_{\theta} \quad \mathrm{PA}+\gamma+\beta \vdash \alpha,
$$

or

$$
\mathrm{PA}+\neg \alpha+\beta \vdash \neg \gamma \text {. }
$$

We also have that $F_{\theta}$ is a maximal element of $E_{\theta} \mathrm{n}$ and therefore $\forall \xi \in F_{\theta} \mathrm{PA}+$ $\neg\left(L \backslash F_{\theta}\right)+\theta+\neg \xi$ is an inconsistent theory (because, if this theory is consistent, the prime filter of all $\forall_{1}$-sentences true in any model of this theory is an element of $E_{\theta}$ properly contained in $F_{\theta}$ ). Therefore,

$$
\forall \xi \in F_{\theta}
$$$$
\exists \rho \in L \backslash F_{\theta}
$$$$
\text { PA }+\theta+\xi \vee \rho .
$$

Let $\alpha \in F^{\prime} \backslash F_{\theta}$. By a result of Solovary (cf. [1, Theorem 2.7]), we know that there is a $\forall_{1}$-sentence $\varphi$, independent of PA $+\theta+\neg \alpha$, such that

(I) $\mathrm{PA}+\theta+\neg \alpha+\varphi$ and PA $+\theta+\neg \alpha$ have the same $\exists_{1}$-theorems,

(II) $\mathrm{PA}+\theta+\neg \alpha+\neg \varphi$ and $\mathrm{PA}+\theta+\neg \alpha$ have the same $\forall_{1}$-theorems. $\varphi \notin F_{\theta}$; because, if $\varphi \in F_{\theta}$ then, by (**), we have

$$
\begin{array}{ll}
\exists \varphi^{\prime} \in L \backslash F_{\theta} \quad & \mathrm{PA}+\theta+\varphi \vee \varphi^{\prime}, \\
& \mathrm{PA}+\theta+\neg \alpha \vdash \varphi \vee \varphi^{\prime}, \\
& \mathrm{PA}+\theta+\neg \alpha+\neg \varphi \vdash \varphi^{\prime}, \\
& \mathrm{PA}+\theta+\neg \alpha \vdash \varphi^{\prime},
\end{array}
$$


and

$$
\mathrm{PA} \vdash \boldsymbol{\theta} \Rightarrow \alpha \vee \varphi^{\prime},
$$

but $\theta \in F_{\theta}, \alpha \vee \varphi^{\prime} \notin F_{\theta}$ and $\theta \leqslant \alpha \vee \varphi^{\prime}$. Contradiction!

If $\varphi \in L \backslash F_{\theta}$, we have by (*)

$$
\begin{array}{ll}
\exists \gamma \in F_{\theta} & \mathrm{PA}+\neg \alpha+\varphi \vdash \neg \gamma, \\
& \mathrm{PA}+\theta+\neg \alpha+\varphi \vdash \neg \gamma, \\
& \mathrm{PA}+\theta+\neg \alpha \vdash \neg \gamma,
\end{array}
$$

and

$$
\mathrm{PA} \vdash \theta \wedge \gamma \Rightarrow \alpha
$$

but $\alpha \notin F_{\theta}, \theta \wedge \gamma \in F_{\theta}$ and $\theta \wedge \gamma \leqslant \alpha$. Contradiction! $F_{\theta}$ has therefore no immediate predecessor.

(iv) Let $B$ be a branch of the $E$-tree containing $F_{\theta} . F_{\theta}$ is a maximal element of $E_{\theta}$, and therefore $F_{\theta}$ is the greatest element of $B$ containing $\theta$. Let $A=\{F \in B \mid \theta \notin F\}$. It is straightforward to check that $F^{\prime}=\cup_{F \in A} F$ is the lowest element of $B$ which does not contain $\theta . F^{\prime}$ is, of course, an immediate successor of $F_{\theta}$.

LEMMA 2.2. If $F$ is any maximal element of the E-tree, then $F$ has no immediate predecessor.

Proof. We use the same kind of argument as in the proof of Lemma 2.1(iii). Let $\theta=1$. (We delete, of course, the requirement "PA $+\neg \theta$ and PA have the same $\forall_{1}$-theorems" which is not used in the proof of Lemma 2.1(iii).) Now $F_{\theta}$ becomes any maximal element of the $E$-tree.

\section{The main result.}

THEOREM 3.1. The E-tree is not homogeneous.

Proof. This is an immediate consequence of Lemmas 2.1 and 2.2, for, in the notation of Lemma 2.1, $F_{\theta}$ and its immediate successor $F^{\prime}$ are neither minimal nor maximal and yet cannot be exchanged by an automorphism of the $E$-tree.

\section{REFERENCES}

1. D. Guaspari, Partially conservative extensions of arithmetic, Trans. Amer. Math. Soc. 254 (1979), 47-68.

2. D. Jensen and A. Ehrenfeucht, Some problem in elementary arithmetics, Fund. Math. 92 (1976), 223-245.

3. D. Misercque, Solutions de deux problèmes posés par H. Simmons, Proc. Logic Meeting (Brussels and Mons, June 2-5, 1980) Bull. Soc. Math. Belg. 33 (1981), 65-72.

4. H. Simmons, Existentially closed models of basic number theory, Logic Colloquium 76, North-Holland, Amsterdam, 1977, pp. 325-369.

Department of Mathematics, University of Brussels, 1050 Brussels, Belgium 